\title{
A SELF-DUALITY OF STRONG STARLIKENESS
}

\author{
ToshiYuki Sugawa
}

To the memory of Professor Nobuyuki Suita

\begin{abstract}
In this note, we will show that a simply connected bounded domain $D$ is strongly starlike of order $\alpha<1$ with respect to the origin if and only if so is $D^{\vee}$, where $D^{\vee}$ is the analytic inversion of the exterior of $D$, namely, $D^{\vee}=\{w \in \mathbf{C}: 1 / w \in \hat{\mathbf{C}} \backslash \bar{D}\}$. This fact neatly explains the relationship between some known properties of strongly starlike domains and provides several new characterizations for those domains.
\end{abstract}

\section{Characterizations of strongly starlike domains}

1.1. Introduction. A subset $E$ of $\mathbf{C}$ with $0 \in E$ is called starlike with respect to 0 if the closed line segment $[0, a]$ between 0 and $a$ is contained in $E$ for each $a \in E$. Similarly, a subset $F$ of the Riemann sphere $\hat{\mathbf{C}}=\mathbf{C} \cup\{\infty\}$ satisfying $\infty \in F$ and $0 \notin F$ is called starlike with respect to $\infty$ if the line segment $[a, \infty]=$ $\{r a: r \in[1,+\infty]\}$ between $a$ and $\infty$ is contained in $F$ for each $a \in F$.

The present research is based on the very simple fact that the following three properties are equivalent:

(i) $E$ is starlike with respect to 0 ,

(ii) $\hat{\mathbf{C}} \backslash E$ is starlike with respect to $\infty$, and

(iii) $E^{*}=\{a \in \mathbf{C}: 1 / a \in \hat{\mathbf{C}} \backslash E\}$ is starlike with respect to 0 . Note that $\left(E^{*}\right)^{*}=E$.

In the following, we will see an analogous result for strongly starlike domains of order $\alpha \in(0,1)$. This idea will enable us to give a unifying proof for some known results scattered in several papers, and moreover, will suggest new geometric characterizations of strong starlikeness.

1.2. Set operations. We begin by defining several operations on the subsets

1991 Mathematics Subject Classification. Primary 30C45; Secondary 30C20.

Key words and phrases. strongly starlike, logarithmic spiral.

The author was partially supported by the Ministry of Education, Grant-in-Aid for Encouragement of Young Scientists, 11740088. A part of this work was carried out during his visit to the University of Helsinki under the exchange programme of scientists between the Academy of Finland and the JSPS.

Received June 9, 2003; revised March 4, 2004. 
of the Riemann sphere $\hat{\mathbf{C}}$. For a subset $E$ of $\hat{\mathbf{C}}$, we set $E^{-1}=I(E)$ and $a \cdot E=$ $M_{a}(E)$ for $a \in \hat{\mathbf{C}}$, where $I$ and $M_{a}$ denote the Möbius transformations defined by $I(z)=1 / z$ and $M_{a}(z)=a z$ for $a \in \hat{\mathbf{C}}$, respectively. Here we define $M_{0} \equiv 0$ and $M_{\infty} \equiv \infty$. We denote by Ext $E$ the exterior of the set $E$ in $\hat{\mathbf{C}}$, i.e., Ext $E=\hat{\mathbf{C}} \backslash \bar{E}$.

We now modify the above operation $E^{*}$ as follows and use it in the sequel. For a domain $D$, we set $D^{\vee}=(\operatorname{Ext} D)^{-1}$. If $D$ is a bounded Jordan domain containing the origin, then so is $D^{\vee}$ and $\left(D^{\vee}\right)^{\vee}=D$.

1.3. Strong starlikeness. Let $\alpha$ be a real constant with $0 \leq \alpha \leq 1$. A holomorphic function $f$ on the unit disk $\mathbf{D}=\{z \in \mathbf{C}:|z|<1\}$ satisfying $f(0)=0$ and $f^{\prime}(0) \neq 0$ is called strongly starlike of order $\alpha$ if $f$ satisfies the condition

$$
\left|\arg \frac{z f^{\prime}(z)}{f(z)}\right| \leq \frac{\alpha \pi}{2} \quad(z \in \mathbf{D} \backslash\{0\}) .
$$

The study of strongly starlike functions was initiated by Stankiewicz [6] and by Brannan and Kirwan [1], independently. Note that a strongly starlike function is starlike in the usual sense, and, in particular, univalent in the unit disk. We remark that the image of the unit disk under the strongly starlike function of order $\alpha$ is a bounded Jordan domain if $\alpha<1$ (see [1]).

A meromorphic function $g$ on the unit disk with the expansion

$$
g(z)=\frac{b_{-1}}{z}+b_{0}+b_{1} z+b_{2} z^{2}+\cdots,
$$

where $b_{-1} \neq 0$, is called meromorphic strongly starlike of order $\alpha$ if the condition

$$
\left|\arg \frac{-z g^{\prime}(z)}{g(z)}\right| \leq \frac{\alpha \pi}{2} \quad(z \in \mathbf{D} \backslash\{0\})
$$

is satisfied. Since $z g^{\prime} / g$ is holomorphic, we know that $g$ never takes 0 as a value. As we can see from the definition, $g$ is meromorphic strongly starlike of order $\alpha$ if and only if the function $1 / g$ is strongly starlike of order $\alpha$.

A proper subdomain $D$ of the complex plane $\mathbf{C}$ containing the origin is said to be strongly starlike of order $\alpha$ with respect to 0 if $D$ is simply connected and if a conformal map $f$ of $\mathbf{D}$ onto $D$ with $f(0)=0$ is strongly starlike of order $\alpha$. Strong starlikeness of order 1 is nothing but the usual starlikeness. If $\alpha<1$, then $D$ is a bounded Jordan domain starlike with respect to the origin, and hence, so is $D^{\vee}$ as we saw in Section 1.1 .

A proper subdomain $D$ of $\hat{\mathbf{C}} \backslash\{0\}$ containing the point at infinity is called strongly starlike of order $\alpha$ with respect to $\infty$ if $D$ is simply connected and if a conformal map $g$ of $\mathbf{D}$ onto $D$ with $g(0)=\infty$ is meromorphic strongly starlike of order $\alpha$. Of course, this is equivalent to the condition that $D^{-1}$ is strongly starlike of order $\alpha$ with respect to 0 .

1.4. Standard domains. We now introduce four standard domains to be considered. Let $\alpha$ be a constant with $0<\alpha<1$. We define $U_{\alpha}$ by 


$$
\begin{aligned}
U_{\alpha} & =\left\{z:\left|2 z \cos (\alpha \pi / 2)-e^{i \alpha \pi / 2}\right|<1 \text { and }\left|2 z \cos (\alpha \pi / 2)-e^{-i \alpha \pi / 2}\right|<1\right\} \\
& =\left\{z:\left|z-\frac{1}{2}\left(1 \pm i \tan \frac{\alpha \pi}{2}\right)\right|<\frac{1}{2 \cos \frac{\alpha \pi}{2}}\right\} .
\end{aligned}
$$

In other words, $U_{\alpha}$ is the convex Jordan domain bounded by two congruent circular arcs forming the angle $\pi(1-\alpha)$ at the intersection points 0 and 1 .

The next one, $V_{\alpha}$, will be defined as the bounded Jordan domain surrounded by the logarithmic spirals $\gamma_{\alpha}=\{\exp ((-\tan (\alpha \pi / 2)+i) \theta): 0 \leq \theta \leq \pi\}$ and $\bar{\gamma}_{\alpha}=\left\{w: \bar{w} \in \gamma_{\alpha}\right\}$. Therefore,

$$
V_{\alpha}=\left\{r e^{i \theta}: \log r<-|\theta| \tan (\alpha \pi / 2), \theta \in[-\pi, \pi]\right\} .
$$

We also use $U_{\alpha}^{-1}$ and $V_{\alpha}^{-1}$. One can easily see that

$$
U_{\alpha}^{-1}=\{w \in \mathbf{C} \backslash\{1\}:|\arg (w-1)|<\alpha \pi / 2\}
$$

and that $V_{\alpha}^{-1}$ is the exterior of the Jordan domain bounded by the logarithmic spirals $\gamma_{\alpha}^{-1}=\{\exp ((\tan (\alpha \pi / 2)-i) \theta): 0 \leq \theta \leq \pi\}$ and $\bar{\gamma}_{\alpha}^{-1}=\left\{w: \bar{w} \in \gamma_{\alpha}^{-1}\right\}$ and satisfies

$$
\text { Ext } V_{\alpha}^{-1}=-e^{\pi \tan (\alpha \pi / 2)} \cdot V_{\alpha}
$$

(see Figure 1).

1.5. Radius function $R_{D}(\theta)$. Let $D$ be a proper subdomain of $\mathbf{C}$ containing the origin. We define the periodic function $R=R_{D}: \mathbf{R} \rightarrow(0,+\infty]$ by

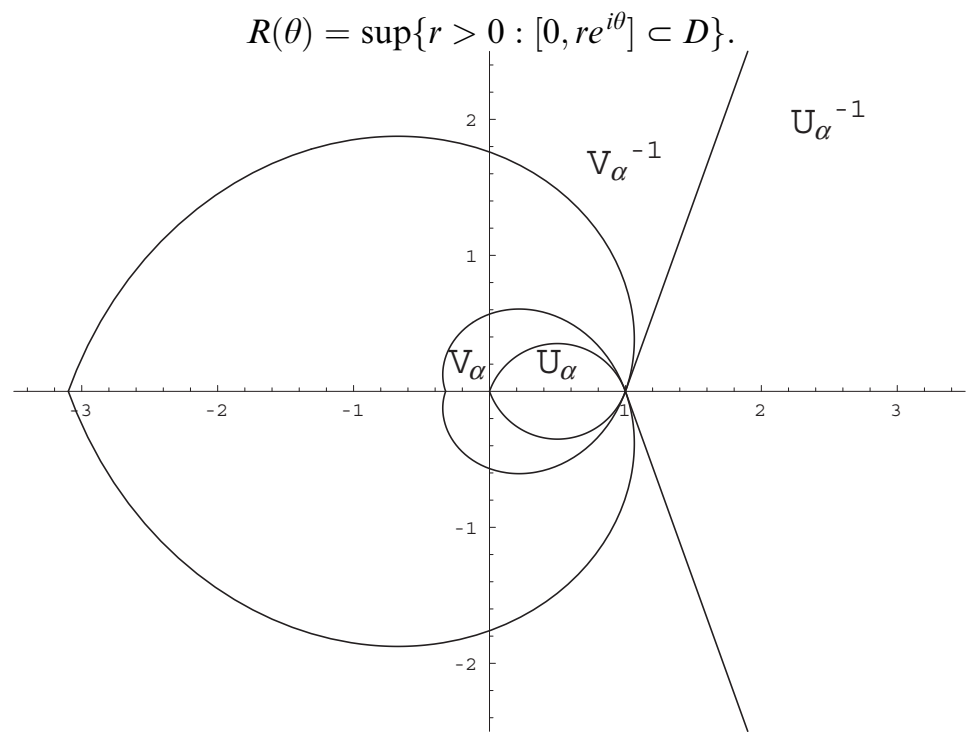

FiguRE 1. $U_{\alpha} \subset V_{\alpha} \subset$ Ext $V_{\alpha}^{-1} \subset \operatorname{Ext} U_{\alpha}^{-1}$ 
Notice that $R$ is lower semi-continuous and reproduces the original domain by the relation $D=\left\{r e^{i \theta}: r<R(\theta)\right\}$ if $D$ is starlike with respect to the origin. For example, $R_{V_{\alpha}}(\theta)=\exp (-|\theta| \tan (\alpha \pi / 2))$ for $\theta \in[-\pi, \pi]$. The following properties are easy to see but constitute a crucial part of the present research.

LEMma 1. If $R_{D}$ is continuous, then $D$ is starlike with respect to the origin. If $R_{D}$ is continuous and bounded, then $D$ is a bounded starlike Jordan domain and the following self-duality relation holds:

$$
R_{D^{\vee}}(\theta)=1 / R_{D}(-\theta) \quad(\theta \in \mathbf{R}) .
$$

1.6. Main result. Our main result is the following, which will be shown in Section 2.

Theorem 1. Let $D$ be a proper subdomain of $\mathbf{C}$ with $0 \in D$ and let $\alpha$ be a constant with $0<\alpha<1$. Then the following seven conditions are equivalent.

(i) $D$ is strongly starlike of order $\alpha$ with respect to the origin.

(ii) Ext $D$ is strongly starlike of order $\alpha$ with respect to $\infty$.

(iii) $w \cdot U_{\alpha} \subset D$ for each $w \in \bar{D}$.

(iv) $w \cdot U_{\alpha}^{-1} \subset$ Ext $D$ for each $w \in \mathbf{C} \backslash D$.

(v) $w \cdot V_{\alpha} \subset D$ for each $w \in \bar{D}$.

(vi) $w \cdot V_{\alpha}^{-1} \subset$ Ext $D$ for each $w \in \mathbf{C} \backslash D$.

(vii) The function $R=R_{D}$ is absolutely continuous and satisfies $\left|R^{\prime} / R\right| \leq$ $\tan (\alpha \pi / 2)$ a.e. in $\mathbf{R}$.

The equivalence of conditions (i), (iv) and (vi) was proved by Stankiewicz [6]. The equivalence of conditions (i) and (iii) is due to Ma and Minda [5]. The implication (i) $\Rightarrow$ (vii) was essentially used by Fait, Krzyż and Zygmunt [3] to show the quasiconformal extendability of strongly starlike functions. The condition (v) is used in [7] to give a lower estimate of the inner radius of univalence for a strongly starlike domain.

\section{Proof of the main theorem}

As we noted, some parts are known. We, however, give the whole proof in order to emphasize the self-duality of strong starlikeness as well as in order to be self-contained.

2.1. Structure of the proof. First we will show the implication (i) $\Rightarrow$ (vii). Then we give a proof for the implications (vii) $\Rightarrow(\mathrm{v}) \Rightarrow$ (iii) $\Rightarrow(\mathrm{i})$. In view of the self-duality relation (4), by applying $D^{\vee}$ instead of $D$ above, we simultaneously have (vii) $\Rightarrow$ (vi) $\Rightarrow$ (iv) $\Rightarrow$ (ii). The condition (ii) for $D$ is equivalent to (i) for $D^{\vee}$. Hence, we immediately obtain the implication (ii) $\Rightarrow$ (i) by the implication (i) $\Rightarrow$ (ii) for the domain $D^{\vee}$.

2.2. A preparatory result. Let $\mathbf{D}_{r}$ denote the disk $\{|z|<r\}$. We need a lemma which appeared in [4] for later use. 
LEMMA 2. Let $f$ be a holomorphic univalent function $f$ on the unit disk with $f(0)=0$. Suppose that the image $D=f(\mathbf{D})$ satisfies the condition (iii) in Theorem 1. Then $f(z) \cdot U_{\alpha} \subset f\left(\mathbf{D}_{r}\right)$ holds for each $|z|=r<1$.

Proof. Fix a point $w \in U_{\alpha}$. Then the function $g(z)=f^{-1}(f(z) w)$ is holomorphic in $\mathbf{D}$ and satisfies $|g(z)|<1, g(0)=0$ and $\left|g^{\prime}(0)\right|=|w|<1$. The Schwarz lemma implies now that $|g(z)|<|z|$ for $z \in \mathbf{D} \backslash\{0\}$, which means $f(z) w \in f\left(\mathbf{D}_{|z|}\right)$.

2.3. (i) implies (vii). We follow the arguments in [1] and [3]. Let $f: \mathbf{D} \rightarrow \mathbf{C}$ be a strongly starlike function of order $\alpha(0<\alpha<1)$. Note that $\left|z f^{\prime}(z)\right| \leq M|q(z)|$, where $M=\sup |f(z)|<\infty$ and $q(z)=z f^{\prime}(z) / f(z)$. Since $q^{1 / \alpha}$ is subordinate to $(1+z) /(1-z)$, Littlewood's Subordination Theorem (see $[2$, Theorem 1.7]) yields that $q^{1 / \alpha}$ is a member of the Hardy space $H^{p}$ for $p<1$. Hence, $q \in H^{p}$ for $p<1 / \alpha$, in particular, $q \in H^{1}$. Note the standard fact that a function $g(z)$ in the Hardy space has the finite limit $g\left(e^{i t}\right)$, called the nontangential limit, when $z$ approaches $e^{i t}$ in any Stolz region for almost all $t$. Since $\left|z f^{\prime}\right| \leq M|q|$, also $f^{\prime} \in H^{1}$ follows. The last assertion implies that $f$ has a continuous extension to $\overline{\mathbf{D}}$ and the extended $f$ is absolutely continuous on the circle $\partial \mathbf{D}$ and the angular limit of $f^{\prime}$ vanishes almost nowhere on $\partial \mathbf{D}$ by the RieszPrivalov theorem (see $[2, \S 3.5]$ ). If we write $f\left(e^{i t}\right)=\rho(t) e^{i \Theta(t)}$, we then observe that $\Theta(t)$ and $\rho(t)$ are absolutely continuous in $t$ and obtain the relation

$$
q\left(e^{i t}\right)=\frac{e^{i t} f^{\prime}\left(e^{i t}\right)}{f\left(e^{i t}\right)}=\Theta^{\prime}(t)-i \frac{\rho^{\prime}(t)}{\rho(t)}
$$

for almost all $t$. By (1), we note that $q\left(e^{i t}\right)$ lies in the closure of the sector $|\arg w|<\alpha \pi / 2$ for almost all $t$. Noting that $f^{\prime}\left(e^{i t}\right) \neq 0$ for almost all $t$, we see that $\Theta^{\prime} \neq 0$ a.e. and $\left|\arg \left(1-i \rho^{\prime} / \Theta^{\prime} \rho\right)\right| \leq \alpha \pi / 2$ a.e., equivalently, $\left|\rho^{\prime} / \Theta^{\prime} \rho\right| \leq$ $\tan (\alpha \pi / 2)$ a.e. Since $\Theta$ is non-decreasing by the starlikeness of $f$, actually $\Theta^{\prime}>0$ holds a.e., and thus, $\Theta: \mathbf{R} \rightarrow \mathbf{R}$ is homeomorphic and $\Theta^{-1}$ preserves sets of linear measure zero. Set $\kappa(\theta)=\rho^{\prime}\left(\Theta^{-1}(\theta)\right) / \Theta^{\prime}\left(\Theta^{-1}(\theta)\right)$. Note that $\kappa(\theta)$ can be defined for almost all $\theta \in \mathbf{R}$ and $\|\kappa\|_{\infty} \leq M \tan (\alpha \pi / 2)<+\infty$. We now consider the absolutely continuous function $F(\theta)=\int_{0}^{\theta} \kappa(x) d x+R(0)$ on $\mathbf{R}$. Since $(F \circ \Theta)^{\prime}=\kappa \circ \Theta \cdot \Theta^{\prime}=\rho^{\prime}$, we have $F \circ \Theta=\rho$, in other words, $F(\theta)=$ $\rho\left(\Theta^{-1}(\theta)\right)=R(\theta)$. The last relation implies that $R$ is absolutely continuous and satisfies $\left|R^{\prime} / R\right|=\left|\rho^{\prime} / \Theta^{\prime} \rho\right| \circ \Theta^{-1} \leq \tan (\alpha \pi / 2)$ a.e.

2.4. (vii) implies (v). Let $w=r e^{i \theta_{0}}$ be an arbitrary point in $\bar{D}$ other than the origin. Set $w_{0}=R\left(\theta_{0}\right) e^{i \theta_{0}}$. Integrating the inequality $R^{\prime} / R \geq$ $-\tan (\alpha \pi / 2)$, we obtain $\log \left(R(\theta) / R\left(\theta_{0}\right)\right) \geq-\left(\theta-\theta_{0}\right) \tan (\alpha \pi / 2)$, i.e., $\quad R(\theta) \geq$ $R\left(\theta_{0}\right) e^{-\left(\theta-\theta_{0}\right) \tan (\alpha \pi / 2)}=R_{w_{0}} \cdot V_{\alpha}(\theta)$ for $\theta \in\left[\theta_{0}, \theta_{0}+\pi\right]$. Similarly we can also show that $R(\theta) \geq R\left(\theta_{0}\right) e^{-\left(\theta_{0}-\theta\right) \tan (\alpha \pi / 2)}=R_{w_{0} \cdot V_{\alpha}}(\theta)$ for $\theta \in\left[\theta_{0}-\pi, \theta_{0}\right]$. Hence, $w \cdot V_{\alpha} \subset$ $w_{0} \cdot V_{\alpha} \subset D$. 
2.5. (v) implies (iii). It is enough to show $U_{\alpha} \subset V_{\alpha}$. For $\theta$ with $|\theta|<\alpha \pi / 2$, we set $r(\theta)=\sup \left\{r>0:\left(0, r e^{i \theta}\right) \subset U_{\alpha}\right\}$. We will show $r(\theta)<$ $R_{V_{\alpha}}(\theta)=e^{-\theta \tan (\alpha \pi / 2)}$ if $0<\theta<\alpha \pi / 2$. An elementary calculation shows that $r(\theta)=\cos (\theta+\alpha \pi / 2) / \cos (\alpha \pi / 2)$. The function $h(\theta)=e^{\theta \tan (\alpha \pi / 2)} \cos (\theta+\alpha \pi / 2) /$ $\cos (\alpha \pi / 2)$ satisfies $h^{\prime}(\theta) / h(\theta)=\tan (\alpha \pi / 2)-\tan (\theta+\alpha \pi / 2)$ for $\theta \in(0, \alpha \pi / 2)$. Therefore $h^{\prime}<0$ and thus $h$ is decreasing. In particular, $h(\theta)<h(0)=1$, which yields the desired inequality.

2.6. (iii) implies (i). Let $f: \mathbf{D} \rightarrow D$ be a conformal map of $\mathbf{D}$ onto $D$ with $f(0)=0$. We use now the method developed by Ma and Minda [5]. Fix a point $z_{0} \in \mathbf{D} \backslash\{0\}$ and set $w_{0}=f\left(z_{0}\right)$. Since the smooth curve $\gamma(t)=f\left(z_{0} e^{i t}\right)$ passes through $w_{0}$ and encloses the domain $w_{0} \cdot U_{\alpha}$ by Lemma 2, we have $\left|\arg \left(\gamma^{\prime}(0) / w_{0}\right)-\pi / 2\right| \leq \alpha \pi / 2$. Since $\gamma^{\prime}(0) / w_{0}=i z_{0} f^{\prime}\left(z_{0}\right) / f\left(z_{0}\right)$, the last inequality gives us $\left|\arg \left(z_{0} f^{\prime}\left(z_{0}\right) / f\left(z_{0}\right)\right)\right| \leq \alpha \pi / 2$.

The program for the proof has now finished.

\section{Concluding remarks}

3.1. The domain $V_{\alpha}$. As is easily seen from the characterization theorem, the domain $V_{\alpha}$ itself is strongly starlike of order $\alpha$. Moreover, the conformal map $g_{\alpha}$ of $\mathbf{D}$ onto $V_{\alpha}$ determined by $g_{\alpha}(0)=0$ and $g_{\alpha}^{\prime}(0)>0$ can be given in the form $k_{\alpha} / k_{\alpha}(1)$, where

$$
k_{\alpha}(z)=z \exp \left[\int_{0}^{z}\left\{\left(\frac{1+\zeta}{1-\zeta}\right)^{\alpha}-1\right\} \frac{d \zeta}{\zeta}\right] .
$$

The function $k_{\alpha}$ plays a role of the Koebe function in the class of normalized strongly starlike functions of order $\alpha$ (see, for example, [5] and its references).

In order to see $g_{\alpha}(\mathbf{D})=V_{\alpha}$, we have only to check

$$
g_{\alpha}(-1)=-\exp (-\pi \tan (\alpha \pi / 2)) .
$$

Indeed, by property (v) in Theorem 1 , we obtain $V_{\alpha} \subset g_{\alpha}(\mathbf{D})$ because $1 \in \partial g_{\alpha}(\mathbf{D})$. On the other hand, by property $(\mathrm{vi})$, we have $g_{\alpha}(\mathbf{D}) \subset \operatorname{Ext}\left(g_{\alpha}(-1) \cdot V_{\alpha}^{-1}\right)$. By (3) and (5), this means $g_{\alpha}(\mathbf{D}) \subset-\exp (-\pi \tan (\alpha \pi / 2)) \cdot$ Ext $V_{\alpha}^{-1}=V_{\alpha}$. Thus we conclude $g_{\alpha}(\mathbf{D})=V_{\alpha}$.

To prove (5), we can use the formula $\int_{0}^{\pi} \cot ^{\alpha}(\theta / 2) d \theta=\pi / \cos (\alpha \pi / 2)$ (see $[1$, (2.4)]). In fact, since we know $g_{\alpha}(-1)<0$, we compute

$$
\begin{aligned}
g_{\alpha}(-1) & =-\left|\exp \left[\int_{1}^{-1}\left(\frac{1+\zeta}{1-\zeta}\right)^{\alpha} \frac{d \zeta}{\zeta}\right]\right|=-\exp \operatorname{Re}\left[i \int_{0}^{\pi}\left(\frac{1+e^{i \theta}}{1-e^{i \theta}}\right)^{\alpha} d \theta\right] \\
& =-\exp \operatorname{Re}\left[i e^{\alpha \pi / 2} \int_{0}^{\pi} \cot ^{\alpha}(\theta / 2) d \theta\right]=-\exp [-\pi \tan (\alpha \pi / 2)]
\end{aligned}
$$


3.2. Examples. In order to check the strong starlikeness of a given domain, condition (vii) in Theorem 1 is probably most convenient. As a simple example, we consider the rectangle $D=\{z=x+i y:|x|<a,|y|<b\}$, where $0<$ $b \leq a$. If $0<\tan \theta<b / a$, we have $R(\theta)=a / \cos \theta$ and $R^{\prime}(\theta) / R(\theta)=\tan \theta$. If $b / a<\tan \theta<\infty$, we have $R(\theta)=b / \sin \theta$ and $R^{\prime}(\theta) / R(\theta)=1 / \tan \theta$. Hence, we see that the essential supremum of $\left|R^{\prime}(\theta) / R(\theta)\right|$ taken over $\theta \in \mathbf{R}$ is $a / b$. This means that $D$ is strongly starlike of order $(2 / \pi) \arctan (a / b)$ with respect to the origin, and the order is best possible. In particular, the square is strongly starlike of order $1 / 2$ with respect to its center.

Another example is the standard domain $U_{\alpha}$. It is intuitively obvious that $U_{\alpha}$ is strongly starlike of order $\alpha$ with respect to the point $1 / 2$. We rigorously check it by using Theorem 1 (vii). As is easily seen, $U_{\alpha}$ is the image of the unit disk under the map $(1+f) / 2$, where $f(z)=\left((1+z)^{\alpha}-(1-z)^{\alpha}\right) /$ $\left((1+z)^{\alpha}+(1-z)^{\alpha}\right)$. We show that $f$ is strongly starlike of order $\alpha$. Let $R=R_{f(\mathbf{D})}$. A simple geometric observation gives us the formula $R(\theta)=$ $R(-\theta)=\sqrt{\tan ^{2}(\alpha \pi / 2) \sin ^{2} \theta+1}-\tan (\alpha \pi / 2) \sin \theta$ for $\theta \in(0, \pi)$. Since $R^{\prime}(\theta) /$ $R(\theta)=-\tan (\alpha \pi / 2) \cos \theta / \sqrt{1+\tan ^{2}(\alpha \pi / 2) \sin ^{2} \theta}$ for $\theta \in(0, \pi)$, we get $\mid R^{\prime}(\theta) /$ $R(\theta)|\leq \tan (\alpha \pi / 2)| \cos \theta \mid \leq \tan (\alpha \pi / 2)$.

3.3. Generalization. As a refinement of the notion of strongly starlike functions of order $\alpha$, we may consider the condition

$$
-\frac{\alpha \pi}{2} \leq \arg \frac{z f^{\prime}(z)}{f(z)} \leq \frac{\beta \pi}{2} \quad(z \in \mathbf{D} \backslash\{0\}),
$$

where $\alpha$ and $\beta$ are constants in $[0,1]$. In this case, by the same argument in Section 2.4, we can see $-\tan (\beta \pi / 2) \leq R^{\prime} / R \leq \tan (\alpha \pi / 2)$ a.e. in R. Thus, we obtain a similar statement to Theorem 1 just by replacing $U_{\alpha}$ and $V_{\alpha}$ by $U_{\alpha, \beta}$ and $V_{\alpha, \beta}$, respectively, where $U_{\alpha, \beta}$ is the intersection of the two disks $\left|2 z \cos (\alpha \pi / 2)-e^{i \alpha \pi / 2}\right|<1$ and $\left|2 z \cos (\pi \beta / 2)-e^{-i \pi \beta / 2}\right|<1$ and $V_{\alpha, \beta}$ is the bounded Jordan domain enclosed by the two logarithmic spirals $\exp ((-\tan (\alpha \pi / 2)+i) \theta)$ $\left(0 \leq \theta \leq \theta_{0}\right) \quad$ and $\exp ((-\tan (\beta \pi / 2)-i) \theta)\left(0 \leq \theta \leq 2 \pi-\theta_{0}\right), \quad$ where $\theta_{0}=$ $2 \pi \tan (\beta \pi / 2) /(\tan (\alpha \pi / 2)+\tan (\beta \pi / 2))$. Note that the corresponding condition to $(\mathrm{v})$ in Theorem 1 was obtained in [4].

\section{REFERENCES}

[ 1 ] D. A. Brannan And W. E. Kirwan, On some classes of bounded univalent functions, J. London Math. Soc. (2) 1 (1969), 431-443.

[2] P. L. Duren, Theory of $H^{p}$ Spaces, Academic Press, New York and London, 1970.

[ 3 ] M. Fait, J. G. Krzyż And J. Zygmunt, Explicit quasiconformal extensions for some classes of univalent functions, Comment. Math. Helv. 51 (1976), 279-285.

[4] A. Lecko and J. Stankiewicz, An internal geometric characterization of some subclasses of starlike functions, XVIth Rolf Nevanlinna Colloquium, Joensuu, 1995 (I. Laine and O. Martio, eds.), Walter de Gruyter, Berlin, 1997, 231-238. 
[5] W. MA AND D. Minda, An internal geometric characterization of strongly starlike functions, Ann. Univ. Mariae Curie-Skłodowska, Sectio A. 45 (1991), 89-97.

[6] J. StankiewicZ, Quelques problèmes extrémaux dans les classes des fonctions $\alpha$-angulairement étoilées, Ann. Univ. Mariae Curie-Skłodowska, Sectio A. 20 (1966), 59-75.

[ 7 ] T. Sugawa, Inner radius of univalence for a strongly starlike domain, Monatsh. Math. 139 (2003), 61-68.

Department of Mathematics

Graduate School of Science

HiROSHIMA UNIVERSITY

Higashi-Hiroshima, 739-8526

JAPAN

E-mail address: sugawa@math.sci.hiroshima-u.ac.jp 